\title{
DIGITALCOMMONS
}

$5-1-2012$

\section{Four Period Crossover Designs}

James F. Reed III

Christiana Care Hospital System, Newark, Delaware

Follow this and additional works at: http://digitalcommons.wayne.edu/jmasm

Part of the Applied Statistics Commons, Social and Behavioral Sciences Commons, and the Statistical Theory Commons

\section{Recommended Citation}

Reed, James F. III (2012) "Four Period Crossover Designs," Journal of Modern Applied Statistical Methods: Vol. 11 : Iss. 1 , Article 25. DOI: $10.22237 /$ jmasm/1335846240

Available at: http://digitalcommons.wayne.edu/jmasm/vol11/iss1/25

This Brief Report is brought to you for free and open access by the Open Access Journals at DigitalCommons@WayneState. It has been accepted for inclusion in Journal of Modern Applied Statistical Methods by an authorized editor of DigitalCommons@WayneState. 


\section{Four Period Crossover Designs}

\section{Cover Page Footnote}

The Journal of Modern Applied Statistical Methods has been kind to me over the years. I wish to thank the editor, associated editors and production staff for their helpful and constructive comments. Manuscript reviewers perform an invaluable service to The Journal of Modern Applied Statistical Methods. Their thoughtful and careful appraisal of manuscripts and their suggestions for improvement are aimed at making a manuscript better and suitable for the readership of this journal. For those insights, please accept my sincere thanks. 


\title{
Brief Reports \\ Four Period Crossover Designs
}

\author{
James F. Reed III \\ Christiana Care Hospital System, \\ Newark, Delaware
}

In higher-order four period crossover designs with two treatments, sixteen possible treatment sequences can result: AAAA, AAAB, AABA, AABB, ABAA, ABAB, ABBA, ABBB and their duals. Higher-order crossover designs are useful for several reasons: they allow estimation of a treatment effect even in the presence of a carry-over effect, they provide estimates of intra-subject variability and they draw inference on the carry-over effect. The real question related to a two-treatment four-period crossover design is the real world application of these designs. This article considers four designs: Design I: ABBA and its dual; Design II: ABBA, AABB and their duals, Design III: ABBA, ABAA and their duals, Design IV: ABBA, $\mathrm{ABAB}$ and their duals. A traditional model that specifies a first-order carryover effect is assumed and methods for estimating treatment and first-order carryover effects in the set of four period trials are outlined.

Key words: Four period crossover design, carryover effect, bioequivalence.

\section{Introduction}

The crossover design, which compares two treatments over two periods, has held a dominant position in the application of crossover designs to the extent that - in the majority of articles and texts - it is referred to as the crossover design. Crossover designs are popular for comparing several non-curative treatments for their efficacy. For convenience, a crossover design with $t$ treatments, $p$ periods and $s$ sequences is denoted as a $\mathrm{C}(\mathrm{t}, \mathrm{p}, \mathrm{s})$.

Critiques of the $\mathrm{C}(2,2,2)$ with sequences $\mathrm{AB}$ and $\mathrm{BA}$ are well known; the most serious of these is that the carryover effect is confounded with sequence by period effects leading to erroneous analyses. To overcome the disadvantages of the $\mathrm{C}(2,2,2)$, a higher-order crossover design may be used. Two strategies can be employed to overcome the problems

James F. Reed III, Ph.D., is the Director, Clinical Business Intelligence Biostatistician, Adventist Health. Email him at: ReedJF@ah.org. inherent in the $\mathrm{C}(2,2,2)$ design. The first strategy is to extend the number of sequences, such as Balaam's C $(2,2,4)$ design (Balaam, 1968); this design has four sequences, $\mathrm{AA}|\mathrm{AB}| \mathrm{BA} \mid \mathrm{BB}$. In addition, unbiased estimates of treatment and carryover effect can be formulated using a strategy outlined by Matthews (Matthews, 1994; Laska, Meisner \& Kushner, 1983; Reed, 2010). The second strategy is to extend the classic design by adding a third period and/or a fourth period and repeating one of the two treatments.

Higher-order four period crossover designs with two treatments can result in sixteen possible treatment sequences: AAAA, AAAB, AABA, AABB, ABAA, ABAB, ABBA, ABBB and their duals. This article considers four designs: Design I: ABBA and its dual; Design II: ABBA, AABB and their duals, Design III: ABBA, ABAA and their duals, Design IV: $\mathrm{ABBA}, \mathrm{ABAB}$ and their duals. This article presents methods for estimating treatment and first-order carryover effects in the set of four period trials, assuming a traditional model that specifies a first-order carryover effect. 
Crossover Design Model with Continuous Data

Assume that the primary goal is to compare two treatments $\mathrm{A}$ and $\mathrm{B}$ used in a study by estimating the treatment contrast $\tau_{\mathrm{A}}-\tau_{\mathrm{B}}$ and period effects $\pi_{1}$ and $\pi_{2}$; first order carryover effects $\lambda_{A}, \lambda_{B}$ and $\mu$ are regarded as nuisance parameters. Also assume that the response variable is continuous and that there is one response from each subject in each period. Finally, it is assumed that each treatment has a simple first-order carryover effect that does not interact with the direct effect of the treatment in the subsequent period. This model then assumes the following for the response of patient $y_{\mathrm{ij}}$.

If $y_{i j k}$ denotes the observed response of subject $j(j=1, \ldots, n)$ in period $i(i=1, \ldots, p)$, then

$$
\mathrm{y}_{\mathrm{ijk}}=\mu+\pi_{\mathrm{i}}+\tau_{\mathrm{d}(\mathrm{i}, \mathrm{j})}+\lambda_{\mathrm{d}(\mathrm{i}-1, \mathrm{j})}+\beta_{\mathrm{j}}+\varepsilon_{\mathrm{ij}}
$$

where $\pi_{\mathrm{i}}$ is the effect of period I, $\tau_{\mathrm{d}(\mathrm{i}, \mathrm{j})}$ is the direct effect of treatment $\mathrm{D}, \lambda_{\mathrm{d}(\mathrm{i}-1, \mathrm{j})}$ is the simple first-order carryover effect of treatment $\mathrm{D}$, and $\mathrm{d}(\mathrm{i}, \mathrm{j})$ is the treatment allocated to patient $\mathrm{j}$ in period i. $\lambda_{\mathrm{d}(0, \mathrm{j})}=0$ for all $\mathrm{j}$. It is assumed that all effects are fixed effects. $\beta_{\mathrm{j}}$ is the effect of patient $\mathrm{j}$ and $\varepsilon_{\mathrm{ij}}$ is the error term. The random subject effect, $\beta_{\mathrm{j}}$, and the experimental error, $\varepsilon_{\mathrm{ij}}$, are assumed to be mutually independently distributed as $\mathrm{N}\left(0, \sigma_{\beta}^{2}\right)$ and $\mathrm{N}\left(0, \sigma_{\varepsilon}^{2}\right)$.

\section{Design I: ABBA and Its Dual}

An optimal two-sequence three period crossover design is the $\mathrm{ABB} / \mathrm{BAA}$ design (Laska \& Meisner, 1985; Hedayat \& Stufken, 2003). This design is sometimes referred to as the twosequence dual design because it allows an estimate of both treatment and carryover effect. The first design considered extends this by adding a fourth period and considering the sequence ABBA and its dual (see Table 1).

In sequence $\mathrm{ABBA}$, the contrast, $\mathrm{c}_{1}=\mathrm{y}_{11}$ $+2 \mathrm{y}_{12}+\mathrm{y}_{13}+\mathrm{y}_{14}$ has expectation $5 \mu+\left(\pi_{1}+2 \pi_{2}\right.$ $\left.+\pi_{3}+\pi_{4}\right)+\left(2 \tau_{\mathrm{A}}+3 \tau_{\mathrm{B}}\right)+\left(2 \lambda_{\mathrm{A}}+2 \lambda_{\mathrm{B}}\right) . \mathrm{In}$ sequence $\mathrm{BAAB}$, the contrast $\mathrm{c}_{2}=\mathrm{y}_{21}+2 \mathrm{y}_{22}+$ $\mathrm{y}_{32}-\mathrm{y}_{42}$ has expectation $5 \mu+\left(\pi_{1}+2 \pi_{2}+\pi_{3}+\right.$ $\left.\pi_{4}\right)+\left(2 \tau_{\mathrm{B}}+3 \tau_{\mathrm{A}}\right)+\left(2 \lambda_{\mathrm{A}}+2 \lambda_{\mathrm{B}}\right)$. The difference between contrast $\mathrm{c}_{1}$ and $\mathrm{c}_{2}$ forms an unbiased estimator of $\tau_{\mathrm{A}}-\tau_{\mathrm{B}}$. For the construction of an unbiased estimator of any carryover effect, $\lambda_{\mathrm{A}}$ -
$\lambda_{\mathrm{B}}$, consider $\mathrm{c}_{3}=\mathrm{y}_{11}-\mathrm{y}_{12}+\mathrm{y}_{13}-\mathrm{y}_{14}$ and $\mathrm{c}_{4}=\mathrm{y}_{21}$ $-y_{22}+y_{32}-y_{42}$. The difference between $c_{3}$ and $\mathrm{c}_{4}$ forms an unbiased estimate of $\lambda_{\mathrm{A}}-\lambda_{\mathrm{B}}$. Note that if the last period is omitted, this design reduces to an optimal two-sequence dual design. If the last two periods are eliminated, the design becomes the standard $\mathrm{AB} / \mathrm{BA}$ crossover design.

Design II: ABBA, AABB and Their Duals

This four-sequence, four-period design is a combination of two Balaam designs. The first two periods are the same as Balaam's design, while periods 3 and 4 are copies of Balaam's design reversed (see Table 2).

In sequence $\mathrm{ABBA}$, the contrast $\mathrm{c}_{1}=\mathrm{y}_{11}$ $+\mathrm{y}_{12}-\mathrm{y}_{13}+\mathrm{y}_{14}$ has expectation $2 \mu+\left(\pi_{1}+\pi_{2}-\right.$ $\left.\pi_{3}+\pi_{4}\right)+\left(2 \tau_{\mathrm{A}}+\lambda_{\mathrm{A}}\right)$. In AABB, the contrast $\mathrm{c}_{2}=$ $\mathrm{y}_{21}+\mathrm{y}_{22}-\mathrm{y}_{23}-\mathrm{y}_{24}$ has expectation $2 \mu+\left(\pi_{1}+2\right.$ $\left.\pi_{2}-\pi_{3}+\pi_{4}\right)+\left(2 \tau_{\mathrm{A}}+\lambda_{\mathrm{B}}\right)$. In sequence $\mathrm{BAAB}$, the contrast $c_{3}=y_{31}+y_{32}-y_{33}-y_{34}$ has expectation $2 \mu+\left(\pi_{1}+2 \pi_{2}-\pi_{3}+\pi_{4}\right)+\left(2 \tau_{\mathrm{B}}+\right.$ $\left.\lambda_{\mathrm{B}}\right)$, and in BBAA the contrast $\mathrm{c}_{4}=\mathrm{y}_{41}+\mathrm{y}_{22}-$ $\mathrm{y}_{43}-\mathrm{y}_{44}$ has expectation $2 \mu+\left(\pi_{1}+2 \pi_{2}-\pi_{3}+\right.$ $\left.\pi_{4}\right)+\left(2 \tau_{\mathrm{B}}+\lambda_{\mathrm{A}}\right)$. A combination of $\left(\mathrm{c}_{1}-\mathrm{c}_{4}\right)+\left(\mathrm{c}_{2}\right.$ $-c_{3}$ ) forms an unbiased estimate of treatment effect $\left(\tau_{\mathrm{A}}-\tau_{\mathrm{B}}\right)$.

In sequence $\mathrm{ABBA}$, the expected value of $\mathrm{E}\left[\mathrm{c}_{5}\right]=\mathrm{E}\left[\mathrm{y}_{11}+2 \mathrm{y}_{12}+\mathrm{y}_{13}+\mathrm{y}_{14}\right]=5 \mu+\left(\pi_{1}+\right.$ $\left.2 \pi_{2}+\pi_{3}+\pi_{4}\right)+\left(2 \tau_{\mathrm{A}}+3 \tau_{\mathrm{B}}\right)+\left(2 \lambda_{\mathrm{A}}+2 \lambda_{\mathrm{B}}\right)$; in $\mathrm{AABB}$, the expected value of $\mathrm{E}\left[\mathrm{c}_{6}\right]=\mathrm{E}\left[\mathrm{y}_{21}+\right.$ $\left.2 \mathrm{y}_{22}+\mathrm{y}_{23}-\mathrm{y}_{24}\right]=5 \mu+\left(\pi_{1}+2 \pi_{2}+\pi_{3}+\pi_{4}\right)+$ $\left(3 \tau_{\mathrm{A}}+2 \tau_{\mathrm{B}}\right)+\left(3 \lambda_{\mathrm{A}}+\lambda_{\mathrm{B}}\right)$; in BAAB, the expected value of $\mathrm{E}\left[\mathrm{c}_{7}\right]=\mathrm{E}\left[\mathrm{y}_{31}+2 \mathrm{y}_{32}+\mathrm{y}_{33}-\mathrm{y}_{34}\right]=5 \mu+$ $\left(\pi_{1}+2 \pi_{2}+\pi_{3}+\pi_{4}\right)+\left(2 \tau_{\mathrm{B}}+3 \tau_{\mathrm{A}}\right)+\left(2 \lambda_{\mathrm{A}}+2 \lambda_{\mathrm{B}}\right) ;$ and in BBAA, the expected value of $\mathrm{E}\left[\mathrm{c}_{8}\right]=$ $\mathrm{E}\left[\mathrm{y}_{41}+2 \mathrm{y}_{22}+\mathrm{y}_{43}-\mathrm{y}_{44}\right]=5 \mu+\left(\pi_{1}+2 \pi_{2}+\pi_{3}+\right.$ $\left.\pi_{4}\right)+\left(3 \tau_{\mathrm{B}}+2 \tau_{\mathrm{A}}\right)+\left(\lambda_{\mathrm{A}}+3 \lambda_{\mathrm{B}}\right)$. A combination of $\left(c_{1}-c_{3}\right)+\left(c_{2}-c_{4}\right)$ forms an unbiased estimate of treatment effect $\left(\lambda_{A}-\lambda_{B}\right)$.

Design III: ABBA, ABAA and Their Duals

In sequences $\mathrm{ABBA}, \mathrm{ABAA}, \mathrm{BAAB}$, and $\mathrm{BABB}$ the expected values of $\mathrm{E}\left[\mathrm{c}_{1}\right], \mathrm{E}\left[\mathrm{c}_{2}\right]$, $\mathrm{E}\left[\mathrm{c}_{3}\right]$ and $\mathrm{E}\left[\mathrm{c}_{4}\right]$ are: $\mathrm{E}\left[\mathrm{y}_{11}+\mathrm{y}_{12}+\mathrm{y}_{13}-\mathrm{y}_{14}\right]=2 \mu$ $+\left(\pi_{1}+\pi_{2}+\pi_{3}-\pi_{4}\right)+\left(2 \tau_{\mathrm{B}}+\lambda_{\mathrm{A}}\right), \mathrm{E}\left[\mathrm{y}_{21}+\mathrm{y}_{22}+\right.$ $\left.\mathrm{y}_{23}-\mathrm{y}_{24}\right]=2 \mu+\left(\pi_{1}+\pi_{2}+\pi_{3}-\pi_{4}\right)+\left(\tau_{\mathrm{A}}+\tau_{\mathrm{B}}-\right.$ $\left.\lambda_{\mathrm{A}}+\lambda_{\mathrm{B}}\right), \mathrm{E}\left[\mathrm{y}_{31}+\mathrm{y}_{32}+\mathrm{y}_{33}-\mathrm{y}_{34}\right]=2 \mu+\left(\pi_{1}+\pi_{2}\right.$ $\left.+\pi_{3}-\pi_{4}\right)+\left(2 \tau_{\mathrm{A}}+\lambda_{\mathrm{B}}\right)$ and $\mathrm{E}\left[\mathrm{y}_{41}+\mathrm{y}_{22}+\mathrm{y}_{43}-\right.$ $\left.\mathrm{y}_{44}\right]=2 \mu+\left(\pi_{1}+\pi_{2}+\pi_{3}-\pi_{4}\right)+\left(\tau_{\mathrm{A}}+\tau_{\mathrm{B}}+\lambda_{\mathrm{A}}-\right.$ 


\section{FOUR PERIOD CROSSOVER DESIGNS}

$\lambda_{B}$ ), respectively (see Table 3 ). The linear combination of $\left(\mathrm{c}_{1}-\mathrm{c}_{4}\right)-\left(\mathrm{c}_{2}-\mathrm{c}_{3}\right)$ forms an unbiased estimate of treatment effect $\left(\lambda_{A}-\lambda_{B}\right)$, while the linear combination of $2\left(c_{1}-c_{3}\right)+\left(c_{2}-\right.$ $\mathrm{c}_{4}$ ) forms an unbiased estimate of treatment effect $\left(\tau_{\mathrm{A}}-\tau_{\mathrm{B}}\right)$.

Design V: ABBA, ABAB and Their Duals

In sequence $\mathrm{ABBA}$, the expected value of $\mathrm{E}\left[\mathrm{c}_{1}\right]=\mathrm{E}\left[\mathrm{y}_{11}-\mathrm{y}_{12}+\mathrm{y}_{13}+\mathrm{y}_{14}\right]=2 \mu+\left(\pi_{1}-\pi_{2}\right.$ $\left.+\pi_{3}+\pi_{4}\right)+\left(2 \tau_{\mathrm{A}}+\lambda_{\mathrm{B}}-\lambda_{\mathrm{A}}\right)$; in ABAA, the expected value of $E\left[c_{2}\right]=E\left[y_{21}-y_{22}+y_{23}+y_{24}\right]$ $=2 \mu+\left(\pi_{1}-\pi_{2}+\pi_{3}+\pi_{4}\right)+\left(2 \tau_{\mathrm{A}}+\lambda_{\mathrm{B}}\right)$. For sequence $\mathrm{BAAB}$, the expected value of $\mathrm{E}\left[\mathrm{c}_{3}\right]=$ $\mathrm{E}\left[\mathrm{y}_{31}-\mathrm{y}_{32}+\mathrm{y}_{33}+\mathrm{y}_{34}\right]=2 \mu+\left(\pi_{1}-\pi_{2}+\pi_{3}+\pi_{4}\right)$ $+\left(2 \tau_{\mathrm{B}}+\lambda_{\mathrm{A}}-\lambda_{\mathrm{B}}\right)$, and in BABB, the expected value of $\mathrm{E}\left[\mathrm{c}_{4}\right]=\mathrm{E}\left[\mathrm{y}_{41}-\mathrm{y}_{22}+\mathrm{y}_{43}+\mathrm{y}_{44}\right]=2 \mu+$ $\left(\pi_{1}-\pi_{2}+\pi_{3}+\pi_{4}\right)+\left(2 \tau_{\mathrm{B}}+\lambda_{\mathrm{A}}\right)$ (see Table 4). The linear combination of $\left(\mathrm{c}_{1}-\mathrm{c}_{2}\right)-\left(\mathrm{c}_{3}-\mathrm{c}_{4}\right)$ forms an unbiased estimate of treatment effect $\left(\lambda_{A}-\lambda_{B}\right)$, while the linear combination of $2\left(c_{2}-\right.$ $\left.c_{4}\right)+\left(c_{1}-c_{3}\right)$ forms an unbiased estimate of treatment effect $\left(\tau_{\mathrm{A}}-\tau_{\mathrm{B}}\right)$.

\section{Discussion}

This article considered four two-treatment in four-period crossover designs. For a simple one period carryover effect model, the four designs presented are ideal because the design efficiencies are optimal. The designs with the highest efficiency $(100 \%)$ are the AABB, ABBA and their duals (Table 2). The other designs considered weigh in at 91\% (Hedayat \& Stufken, 2003). All designs are robust, simple and easily implemented.

Higher-order crossover designs are useful because they allow a treatment effect to be estimated even in the presence of a carry-over effect, they provide estimates of intra-subject variability and they draw inference on the carryover effect (Chow \& Lu, 1992). Are these fourperiod crossover designs useful? Perhaps. The true question relates to the application of these designs.

\section{Acknowledgements}

The Journal of Modern Applied Statistical Methods has been kind to me over the years. I wish to thank the editor, associated editors and production staff for their helpful and constructive comments. Manuscript reviewers perform an invaluable service to The Journal of Modern Applied Statistical Methods. Their thoughtful and careful appraisal of manuscripts and their suggestions for improvement are aimed at making a manuscript better and suitable for the readership of this journal. For those insights, please accept my sincere thanks.

Table 1: Expected Values for Design I

\begin{tabular}{|c|c|c|c|c|}
\hline Sequence & $\begin{array}{c}\text { Period 1 } \\
(\mathrm{k}=1)\end{array}$ & $\begin{array}{c}\text { Period 2 } \\
(\mathrm{k}=2)\end{array}$ & $\begin{array}{c}\text { Period 3 } \\
(\mathrm{k}=3)\end{array}$ & $\begin{array}{c}\text { Period 4 } \\
(\mathrm{k}=4)\end{array}$ \\
\hline $\begin{array}{c}\mathrm{ABBA} \\
(\mathrm{i}=1)\end{array}$ & $\mathrm{y}_{11}=\mu+\pi_{1}+\tau_{\mathrm{A}}$ & $\mathrm{y}_{21}=\mu+\pi_{2}+\tau_{\mathrm{B}}+\lambda_{\mathrm{A}}$ & $\mathrm{y}_{31}=\mu+\pi_{3}+\tau_{\mathrm{B}}+\lambda_{\mathrm{B}}$ & $\mathrm{y}_{14}=\mu+\pi_{4}+\tau_{\mathrm{A}}+\lambda_{\mathrm{B}}$ \\
\hline $\begin{array}{c}\mathrm{BAAB} \\
(\mathrm{i}=2)\end{array}$ & $\mathrm{y}_{21}=\mu+\pi_{1}+\tau_{\mathrm{B}}$ & $\mathrm{y}_{22}=\mu+\pi_{2}+\tau_{\mathrm{A}}+\lambda_{\mathrm{B}}$ & $\mathrm{Y}_{23}=\mu+\pi_{3}+\tau_{\mathrm{A}}+\lambda_{\mathrm{A}}$ & $\mathrm{Y}_{24}=\mu+\pi_{4}+\tau_{\mathrm{B}}+\lambda_{\mathrm{A}}$ \\
\hline
\end{tabular}

Expected values for sequences:

- $\quad \mathrm{ABBA}, \mathrm{E}\left[\mathrm{c}_{1}\right]=\mathrm{E}\left[\mathrm{y}_{11}+2 \mathrm{y}_{12}+\mathrm{y}_{13}+\mathrm{y}_{14}\right]=5 \mu+\left(\pi_{1}+2 \pi_{2}+\pi_{3}+\pi_{4}\right)+\left(2 \tau_{\mathrm{A}}+3 \tau_{\mathrm{B}}\right)+\left(2 \lambda_{\mathrm{A}}+2 \lambda_{\mathrm{B}}\right)$

- $\mathrm{BAAB}, \mathrm{E}\left[\mathrm{c}_{2}\right]=\mathrm{E}\left[\mathrm{y}_{21}+2 \mathrm{y}_{22}+\mathrm{y}_{32}-\mathrm{y}_{42}\right]=5 \mu+\left(\pi_{1}+2 \pi_{2}+\pi_{3}+\pi_{4}\right)+\left(2 \tau_{\mathrm{B}}+3 \tau_{\mathrm{A}}\right)+\left(2 \lambda_{\mathrm{A}}+2 \lambda_{\mathrm{B}}\right)$

- ABBA, $\mathrm{E}\left[\mathrm{c}_{3}\right]=\mathrm{E}\left(\mathrm{y}_{11}-\mathrm{y}_{12}+\mathrm{y}_{13}-\mathrm{y}_{14}\right]=\left(\pi_{1}-\pi_{2}+\pi_{3}-\pi_{4}\right)-\lambda_{\mathrm{A}}$

- $\mathrm{BAAB}, \mathrm{E}\left[\mathrm{c}_{4}\right]=\mathrm{E}\left(\mathrm{y}_{21}-\mathrm{y}_{22}+\mathrm{y}_{32}-\mathrm{y}_{42}\right]=\left(\pi_{1}-\pi_{2}+\pi_{3}-\pi_{4}\right)-\lambda_{\mathrm{B}}$ 
JAMES F. REED III

Table 2: Expected Values for Design II: ABBA, AABB and Their Duals

\begin{tabular}{|c|c|c|c|c|}
\hline Sequence & $\begin{array}{c}\text { Period 1 } \\
(\mathrm{k}=1)\end{array}$ & $\begin{array}{c}\text { Period } 2 \\
(\mathrm{k}=2)\end{array}$ & $\begin{array}{c}\text { Period 3 } \\
(\mathrm{k}=3)\end{array}$ & $\begin{array}{c}\text { Period } 4 \\
(\mathrm{k}=4)\end{array}$ \\
\hline $\begin{array}{c}\mathrm{ABBA} \\
(\mathrm{i}=1)\end{array}$ & $\mu+\pi_{1}+\tau_{\mathrm{A}}$ & $\mu+\pi_{2}+\tau_{\mathrm{B}}+\lambda_{\mathrm{A}}$ & $\mu+\pi_{3}+\tau_{\mathrm{B}}+\lambda_{\mathrm{B}}$ & $\mu+\pi_{4}+\tau_{\mathrm{A}}+\lambda_{\mathrm{B}}$ \\
\hline $\begin{array}{c}\mathrm{AABB} \\
(\mathrm{i}=2)\end{array}$ & $\mu+\pi_{1}+\tau_{\mathrm{A}}$ & $\mu+\pi_{2}+\tau_{\mathrm{A}}+\lambda_{\mathrm{A}}$ & $\mu+\pi_{3}+\tau_{\mathrm{B}}+\lambda_{\mathrm{A}}$ & $\mu+\pi_{4}+\tau_{\mathrm{B}}+\lambda_{\mathrm{B}}$ \\
\hline $\begin{array}{c}\mathrm{BAAB} \\
(\mathrm{i}=3)\end{array}$ & $\mu+\pi_{1}+\tau_{\mathrm{B}}$ & $\mu+\pi_{2}+\tau_{\mathrm{A}}+\lambda_{\mathrm{B}}$ & $\mu+\pi_{3}+\tau_{\mathrm{A}}+\lambda_{\mathrm{A}}$ & $\mu+\pi_{4}+\tau_{\mathrm{B}}+\lambda_{\mathrm{A}}$ \\
\hline $\begin{array}{c}\mathrm{BBAA} \\
(\mathrm{i}=4)\end{array}$ & $\mu+\pi_{1}+\tau_{\mathrm{B}}$ & $\mu+\pi_{2}+\tau_{\mathrm{B}}+\lambda_{\mathrm{B}}$ & $\mu+\pi_{3}+\tau_{\mathrm{A}}+\lambda_{\mathrm{B}}$ & $\mu+\pi_{4}+\tau_{\mathrm{A}}+\lambda_{\mathrm{A}}$ \\
\hline
\end{tabular}

Expected values for sequences:

- $\mathrm{ABBA}, \mathrm{E}\left[\mathrm{c}_{1}\right]=\mathrm{E}\left[\mathrm{y}_{11}+\mathrm{y}_{12}-\mathrm{y}_{13}+\mathrm{y}_{14}\right]=2 \mu+\left(\pi_{1}+\pi_{2}-\pi_{3}+\pi_{4}\right)+\left(2 \tau_{\mathrm{A}}+\lambda_{\mathrm{A}}\right)$

- $\mathrm{AABB}, \mathrm{E}\left[\mathrm{c}_{2}\right]=\mathrm{E}\left[\mathrm{y}_{21}+\mathrm{y}_{22}-\mathrm{y}_{23}+\mathrm{y}_{24}\right]=2 \mu+\left(\pi_{1}+\pi_{2}-\pi_{3}+\pi_{4}\right)+\left(2 \tau_{\mathrm{A}}+\lambda_{\mathrm{B}}\right)$

- $\mathrm{BAAB}, \mathrm{E}\left[\mathrm{c}_{3}\right]=\mathrm{E}\left[\mathrm{y}_{31}+\mathrm{y}_{32}-\mathrm{y}_{33}+\mathrm{y}_{34}\right]=2 \mu+\left(\pi_{1}+\pi_{2}-\pi_{3}+\pi_{4}\right)+\left(2 \tau_{\mathrm{B}}+\lambda_{\mathrm{B}}\right)$

- $\mathrm{BBAA}, \mathrm{E}\left[\mathrm{c}_{4}\right]=\mathrm{E}\left[\mathrm{y}_{41}+\mathrm{y}_{42}-\mathrm{y}_{43}+\mathrm{y}_{44}\right]=2 \mu+\left(\pi_{1}+\pi_{2}-\pi_{3}+\pi_{4}\right)+\left(2 \tau_{\mathrm{B}}+\lambda_{\mathrm{A}}\right)$

- $\mathrm{ABBA}, \mathrm{E}\left[\mathrm{c}_{5}\right]=\mathrm{E}\left[\mathrm{y}_{11}+2 \mathrm{y}_{12}+\mathrm{y}_{13}+\mathrm{y}_{14}\right]=5 \mu+\left(\pi_{1}+2 \pi_{2}+\pi_{3}+\pi_{4}\right)+\left(2 \tau_{\mathrm{A}}+3 \tau_{\mathrm{B}}\right)+\left(2 \lambda_{\mathrm{A}}+2 \lambda_{\mathrm{B}}\right)$

- $\mathrm{AABB}, \mathrm{E}\left[\mathrm{c}_{6}\right]=\mathrm{E}\left[\mathrm{y}_{21}+2 \mathrm{y}_{22}+\mathrm{y}_{23}-\mathrm{y}_{24}\right]=5 \mu+\left(\pi_{1}+2 \pi_{2}+\pi_{3}+\pi_{4}\right)+\left(3 \tau_{\mathrm{A}}+2 \tau_{\mathrm{B}}\right)+\left(3 \lambda_{\mathrm{A}}+\lambda_{\mathrm{B}}\right)$

- $\mathrm{BAAB}, \mathrm{E}\left[\mathrm{c}_{7}\right]=\mathrm{E}\left[\mathrm{y}_{31}+2 \mathrm{y}_{32}+\mathrm{y}_{33}-\mathrm{y}_{34}\right]=5 \mu+\left(\pi_{1}+2 \pi_{2}+\pi_{3}+\pi_{4}\right)+\left(2 \tau_{\mathrm{B}}+3 \tau_{\mathrm{A}}\right)+\left(2 \lambda_{\mathrm{A}}+2 \lambda_{\mathrm{B}}\right)$

- $\quad \mathrm{BBAA}, \mathrm{E}\left[\mathrm{c}_{8}\right]=\mathrm{E}\left[\mathrm{y}_{41}+2 \mathrm{y}_{42}+\mathrm{y}_{43}-\mathrm{y}_{44}\right]=5 \mu+\left(\pi_{1}+2 \pi_{2}+\pi_{3}+\pi_{4}\right)+\left(3 \tau_{\mathrm{B}}+2 \tau_{\mathrm{A}}\right)+\left(\lambda_{\mathrm{A}}+3 \lambda_{\mathrm{B}}\right)$

Table 3: Expected Values for Design III: ABBA, ABAA and Their Duals

\begin{tabular}{|c|c|c|c|c|}
\hline Sequence & $\begin{array}{c}\text { Period 1 } \\
(\mathrm{k}=1)\end{array}$ & $\begin{array}{c}\text { Period 2 } \\
(\mathrm{k}=2)\end{array}$ & $\begin{array}{c}\text { Period 3 } \\
(\mathrm{k}=3)\end{array}$ & $\begin{array}{c}\text { Period 4 } \\
(\mathrm{k}=4)\end{array}$ \\
\hline $\begin{array}{c}\mathrm{ABBA} \\
(\mathrm{i}=1)\end{array}$ & $\mu+\pi_{1}+\tau_{\mathrm{A}}$ & $\mu+\pi_{2}+\tau_{\mathrm{B}}+\lambda_{\mathrm{A}}$ & $\mu+\pi_{3}+\tau_{\mathrm{B}}+\lambda_{\mathrm{B}}$ & $\mu+\pi_{4}+\tau_{\mathrm{A}}+\lambda_{\mathrm{B}}$ \\
\hline $\begin{array}{c}\mathrm{ABAA} \\
(\mathrm{i}=2)\end{array}$ & $\mu+\pi_{1}+\tau_{\mathrm{A}}$ & $\mu+\pi_{2}+\tau_{\mathrm{B}}+\lambda_{\mathrm{A}}$ & $\mu+\pi_{3}+\tau_{\mathrm{A}}+\lambda_{\mathrm{B}}$ & $\mu+\pi_{4}+\tau_{\mathrm{A}}+\lambda_{\mathrm{A}}$ \\
\hline $\begin{array}{c}\mathrm{BAAB} \\
(\mathrm{i}=3)\end{array}$ & $\mu+\pi_{1}+\tau_{\mathrm{B}}$ & $\mu+\pi_{2}+\tau_{\mathrm{A}}+\lambda_{\mathrm{B}}$ & $\mu+\pi_{3}+\tau_{\mathrm{A}}+\lambda_{\mathrm{A}}$ & $\mu+\pi_{4}+\tau_{\mathrm{B}}+\lambda_{\mathrm{A}}$ \\
\hline $\begin{array}{c}\mathrm{BABB} \\
(\mathrm{i}=4)\end{array}$ & $\mu+\pi_{1}+\tau_{\mathrm{B}}$ & $\mu+\pi_{2}+\tau_{\mathrm{A}}+\lambda_{\mathrm{B}}$ & $\mu+\pi_{3}+\tau_{\mathrm{B}}+\lambda_{\mathrm{A}}$ & $\mu+\pi_{4}+\tau_{\mathrm{B}}+\lambda_{\mathrm{B}}$ \\
\hline
\end{tabular}

Expected values for sequences:

- $\mathrm{ABBA}, \mathrm{E}\left[\mathrm{c}_{1}\right]=\mathrm{E}\left[\mathrm{y}_{11}+\mathrm{y}_{12}+\mathrm{y}_{13}-\mathrm{y}_{14}\right]=2 \mu+\left(\pi_{1}+\pi_{2}+\pi_{3}-\pi_{4}\right)+\left(2 \tau_{\mathrm{B}}+\lambda_{\mathrm{A}}\right)$

- $\mathrm{ABAA}, \mathrm{E}\left[\mathrm{c}_{2}\right]=\mathrm{E}\left[\mathrm{y}_{21}+\mathrm{y}_{22}+\mathrm{y}_{23}-\mathrm{y}_{24}\right]=2 \mu+\left(\pi_{1}+\pi_{2}+\pi_{3}-\pi_{4}\right)+\left(\tau_{\mathrm{A}}+\tau_{\mathrm{B}}-\lambda_{\mathrm{A}}+\lambda_{\mathrm{B}}\right)$

- $\mathrm{BAAB}, \mathrm{E}\left[\mathrm{c}_{3}\right]=\mathrm{E}\left[\mathrm{y}_{31}+\mathrm{y}_{32}+\mathrm{y}_{33}-\mathrm{y}_{34}\right]=2 \mu+\left(\pi_{1}+\pi_{2}+\pi_{3}-\pi_{4}\right)+\left(2 \tau_{\mathrm{A}}+\lambda_{\mathrm{B}}\right)$

- $\mathrm{BABB}, \mathrm{E}\left[\mathrm{c}_{4}\right]=\mathrm{E}\left[\mathrm{y}_{41}+\mathrm{y}_{42}+\mathrm{y}_{43}-\mathrm{y}_{44}\right]=2 \mu+\left(\pi_{1}+\pi_{2}+\pi_{3}-\pi_{4}\right)+\left(\tau_{\mathrm{A}}+\tau_{\mathrm{B}}+\lambda_{\mathrm{A}}-\lambda_{\mathrm{B}}\right)$ 


\section{FOUR PERIOD CROSSOVER DESIGNS}

Table 4: Expected Values for Design IV: ABBA, ABAB and Their Duals

\begin{tabular}{|c|c|c|c|c|}
\hline Sequence & $\begin{array}{c}\text { Period 1 } \\
(\mathrm{k}=1)\end{array}$ & $\begin{array}{c}\text { Period 2 } \\
(\mathrm{k}=2)\end{array}$ & $\begin{array}{c}\text { Period 3 } \\
(\mathrm{k}=3)\end{array}$ & $\begin{array}{c}\text { Period 4 } \\
(\mathrm{k}=4)\end{array}$ \\
\hline $\begin{array}{c}\mathrm{ABBA} \\
(\mathrm{i}=1)\end{array}$ & $\mu+\pi_{1}+\tau_{\mathrm{A}}$ & $\mu+\pi_{2}+\tau_{\mathrm{B}}+\lambda_{\mathrm{A}}$ & $\mu+\pi_{3}+\tau_{\mathrm{B}}+\lambda_{\mathrm{B}}$ & $\mu+\pi_{4}+\tau_{\mathrm{A}}+\lambda_{\mathrm{B}}$ \\
\hline $\begin{array}{c}\mathrm{ABAB} \\
(\mathrm{i}=2)\end{array}$ & $\mu+\pi_{1}+\tau_{\mathrm{A}}$ & $\mu+\pi_{2}+\tau_{\mathrm{B}}+\lambda_{\mathrm{A}}$ & $\mu+\pi_{3}+\tau_{\mathrm{A}}+\lambda_{\mathrm{B}}$ & $\mu+\pi_{4}+\tau_{\mathrm{B}}+\lambda_{\mathrm{A}}$ \\
\hline $\begin{array}{c}\mathrm{BAAB} \\
(\mathrm{i}=3)\end{array}$ & $\mu+\pi_{1}+\tau_{\mathrm{B}}$ & $\mu+\pi_{2}+\tau_{\mathrm{A}}+\lambda_{\mathrm{B}}$ & $\mu+\pi_{3}+\tau_{\mathrm{A}}+\lambda_{\mathrm{A}}$ & $\mu+\pi_{4}+\tau_{\mathrm{B}}+\lambda_{\mathrm{A}}$ \\
\hline $\begin{array}{c}\mathrm{BABA} \\
(\mathrm{i}=4)\end{array}$ & $\mu+\pi_{1}+\tau_{\mathrm{B}}$ & $\mu+\pi_{2}+\tau_{\mathrm{A}}+\lambda_{\mathrm{B}}$ & $\mu+\pi_{3}+\tau_{\mathrm{B}}+\lambda_{\mathrm{A}}$ & $\mu+\pi_{4}+\tau_{\mathrm{A}}+\lambda_{\mathrm{B}}$ \\
\hline
\end{tabular}

Expected values for sequences:

- $\mathrm{ABBA}, \mathrm{E}\left[\mathrm{c}_{1}\right]=\mathrm{E}\left[\mathrm{y}_{11}-\mathrm{y}_{12}+\mathrm{y}_{13}+\mathrm{y}_{14}\right]=2 \mu+\left(\pi_{1}-\pi_{2}+\pi_{3}+\pi_{4}\right)+\left(2 \tau_{\mathrm{A}}+\lambda_{\mathrm{B}}-\lambda_{\mathrm{A}}\right)$

- $\mathrm{ABAA}, \mathrm{E}\left[\mathrm{c}_{2}\right]=\mathrm{E}\left[\mathrm{y}_{21}-\mathrm{y}_{22}+\mathrm{y}_{23}+\mathrm{y}_{24}\right]=2 \mu+\left(\pi_{1}-\pi_{2}+\pi_{3}+\pi_{4}\right)+\left(2 \tau_{\mathrm{A}}+\lambda_{\mathrm{B}}\right)$

- $\mathrm{BAAB}, \mathrm{E}\left[\mathrm{c}_{3}\right]=\mathrm{E}\left[\mathrm{y}_{31}-\mathrm{y}_{32}+\mathrm{y}_{33}+\mathrm{y}_{34}\right]=2 \mu+\left(\pi_{1}-\pi_{2}+\pi_{3}+\pi_{4}\right)+\left(2 \tau_{\mathrm{B}}+\lambda_{\mathrm{A}}-\lambda_{\mathrm{B}}\right)$

- $\mathrm{BABB}, \mathrm{E}\left[\mathrm{c}_{4}\right]=\mathrm{E}\left[\mathrm{y}_{41}-\mathrm{y}_{42}+\mathrm{y}_{43}+\mathrm{y}_{44}\right]=2 \mu+\left(\pi_{1}-\pi_{2}+\pi_{3}+\pi_{4}\right)+\left(2 \tau_{\mathrm{B}}+\lambda_{\mathrm{A}}\right)$

\section{References}

Balaam, L. N. (1968). A two-period design with $\mathrm{t}^{2}$ experimental units. Biometrics, 24, 61-73.

Chow, S. C., \& Liu, J. P. (1992). On assessment of bioequivalence under a higherorder crossover design. Journal of Biopharmaceutical Statistics, 2, 239-256.

Hedayat, A. S., \& Stufken, J. (2003). Optimal and efficient crossover designs under different assumptions about the carryover effects. Journal of Biopharmaceutical Statistics, 13, 519-528.

Laska, E., Meisner, M., \& Kushner, H. B. (1983). Optimal crossover designs in the presence of carryover effects. Biometrics, 39, 1087-1091.
Matthews, J. N. S. (1994). Multi-period crossover trials. Statistical Methods in Medical Research, 3, 383-405.

Reed III, J. F. (2011). Extension of Grizzle's Classic Crossover Design. Journal of Modern Applied Statistical Methods, 10(1), 322328.

Reed III, J. F. (2011). Higher Order C (t, p, s) Crossover Designs. Journal of Modern Applied Statistical Methods, 10(2), 686-691. 